\title{
MICROACOUSTIC SENSORS WITH INTEGRATED CARBON NANOTUBES FOR HIGH VOC SENSITIVITY
}

\author{
Teona Mirea, Jimena Olivares, and Marta Clement
}

\begin{abstract}
Electroacoustic resonators have proved to be appropriate candidates as VOC sensors owing to their high mass sensitivity. However, one of their drawbacks is their sensitivity to other external factors like the humidity. Here we propose an AlN-based solidly mounted resonator for VOC detection and humidity discrimination. This ability is achieved by defining different areas within the same device with different resonant characteristics by integrating carbon nanotubes (CNTs) in part of the exposed surface while leaving the other bare. While CNTs act as sensing elements adsorbing VOC molecules, hence originating larger frequency shifts, the bare zone is affected only by the ambient humidity.
\end{abstract}

\section{KEYWORDS}

Solidly mounted resonators; volatile organic compound; carbon nanotubes.

\section{INTRODUCTION}

Volatile organic compounds (VOCs) are among the dangerous pollutants that can degrade the air quality and be harmful for humans. They can become toxic even at low concentrations if long exposure times are allowed. Therefore, fast and cheap VOC sensors with high resolution (i.e. able to detect very small amounts) are desirable to ensure safeness in many environments. There exist a large variety of sensors used for this purpose [1]. Among them, acoustic devices have attracted the attention due to their high mass sensitivity. Particularly, surface acoustic wave (SAW) devices are the most commonly used [2], [3]. To boost resolution and mass sensitivity, thin film electroacoustic devices appear as an appropriate alternative owing to the high resolution the can achieve even in the pg range[4]. The work principle of electroacoustic sensors is the shift in their resonant frequencies upon trapped or absorbed molecules to their surface (i.e. they translate mass to frequency shifts). To be selective or discriminative among different VOCs, all of the above-mentioned sensing technologies must include a specifically functionalized sensing layer. This can be achieved by direct functionalization of the sensor surface [5] or by adding polymers or specific material capable absorb the target molecules. In this context carbon nanotubes (CNTs) have appeared as good candidates owing to their high reactivity with VOCs [6] and the large surface area they provide for target binding. CNTs have been typically used in their dispersion form mixed in composites [7]; however, their vertically-aligned structure provides larger exposed areas for binding and increased control on their morphology. Namely, they can be selectively grown by patterning a thin catalyst on the sensor with accurate tube length and separation.

One of the main problems of acoustic wave sensors is their sensitivity to many external factors, namely temperature, humidity, pressure, that hampers the sensitivity to the targeted physical magnitude, in this case mass. In the case of VOC detection one of the main issues is the influence of the ambient humidity while detecting VOC molecules.

We propose in this work an AlN-based solidly mounted resonator (SMR) with integrated CNT forest able to discriminate between mass and humidity detection.

\section{EXPERIMENTAL}

SMRs are based on a piezoelectric AlN active layer sandwiched between two electrodes (Ir, W or Mo) and deposited on top of an acoustic reflector. This last is made of five alternating layers of high (Mo) and low $\left(\mathrm{SiO}_{2}\right)$ acoustic impedance materials, meant to prevent acoustic energy dissipation to the substrate (Figure 1). The CNT forests were grown directly on the top electrode of the devices. To do so, we adapted the devices to hold up to $650^{\circ} \mathrm{C}$, the needed temperature to grow CNT forests [8]. Additionally, we used a two-stage method to grow CNTs on metallic substrates (namely the top electrode) [9]. Among the different CNT forests explored, $5 \mu \mathrm{m}$ long CNTs (Figure 2) appeared to be the most appropriated in order to boost VOC sensitivity without degrading the resonator performance [10].

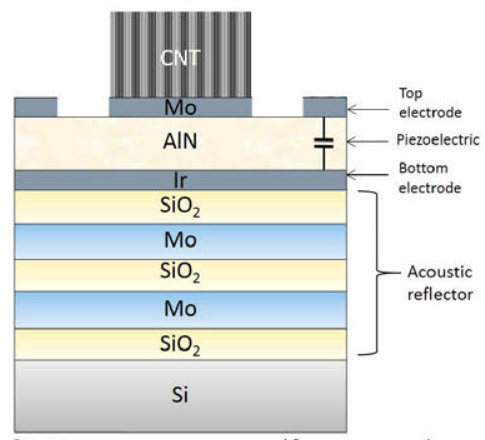

Figure. 1: SMR cross section (front view)

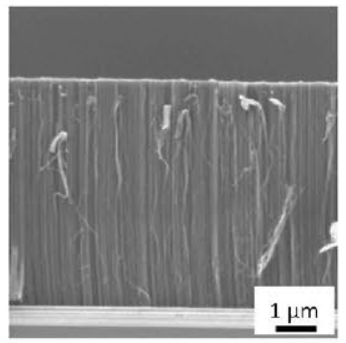

Figure 2: SEM image of grown CNT forests. 
To be able to discriminate between mass and humidity detection, we performed a top electrode pad regrowth in order to define three different sensing areas (see figure 3). Each of the zones displayed a particular resonant frequency (figure 4) being two of them covered with CNTs ( 1 and 3 ). Resonant frequencies 1 and 2 ( $f_{1}$ and $f_{2}$ ) are lower than $f_{3}$ due to the increased thickness of the electrode. Additionally, $\mathrm{f}_{1}$ is slightly lower than $\mathrm{f}_{2}$ due to the presence of CNTs.

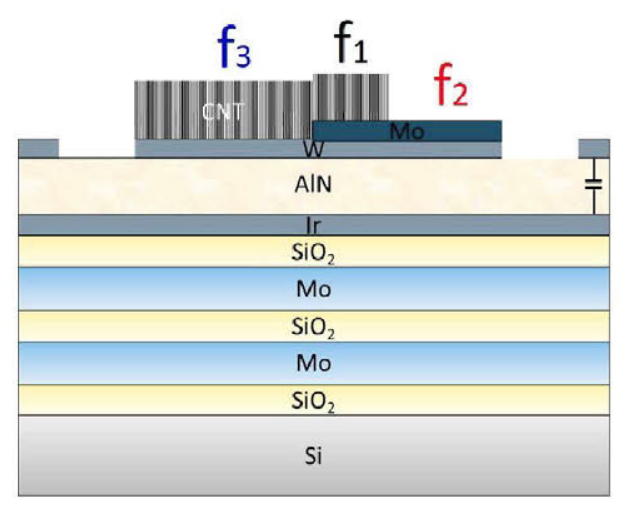

Side cross section view

(a)

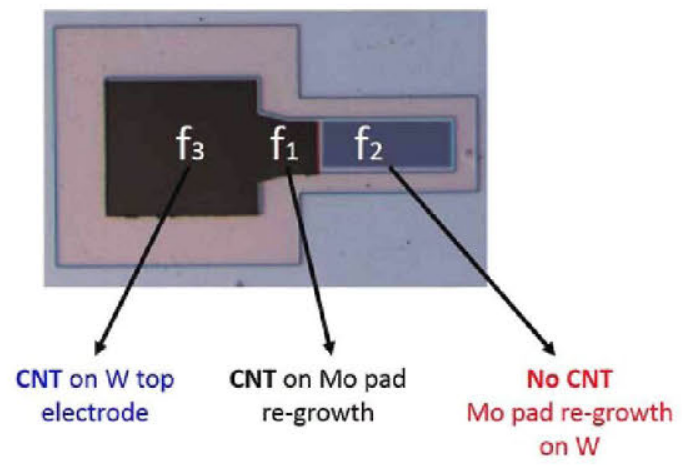

(b)

Figure 3: a) Sketch of the cross section of the SMR and b) picture showing a top view $p f$ the SMR displaying the three different areas exposed to the VOCs. Areas 1 and 3 are covered with CNTs whereas area 2 corresponds to the bare SMR with a WMo top electrode.

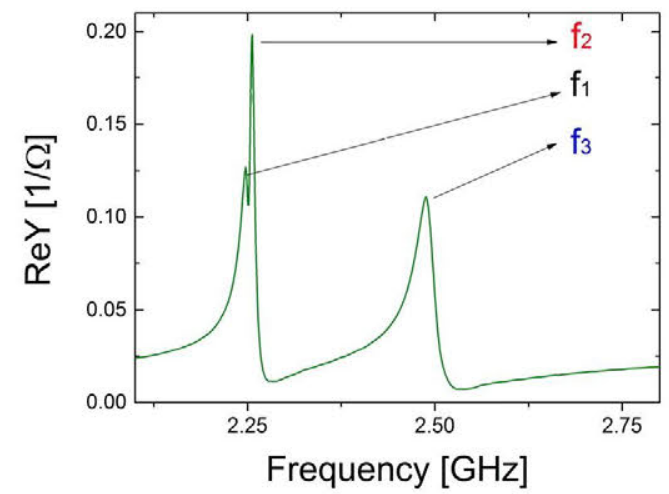

Figure 4: Real part of the admittance of the device highlighting the three resonances corresponding to the three different areas.
A proof-of-concept of VOC detection with the SMRs was carry out with ethanol. $\mathrm{N}_{2}$ was used both as purging and carrier gas, as shown in figure 5. Different ethanol concentrations were obtained by modifying the flow of $\mathrm{N}_{2}$ bubbling in ethanol. The vapors were fed to a small plastic chamber containing the device. Real time frequency monitoring was achieved with an RF probe passing through the plastic enclosure.

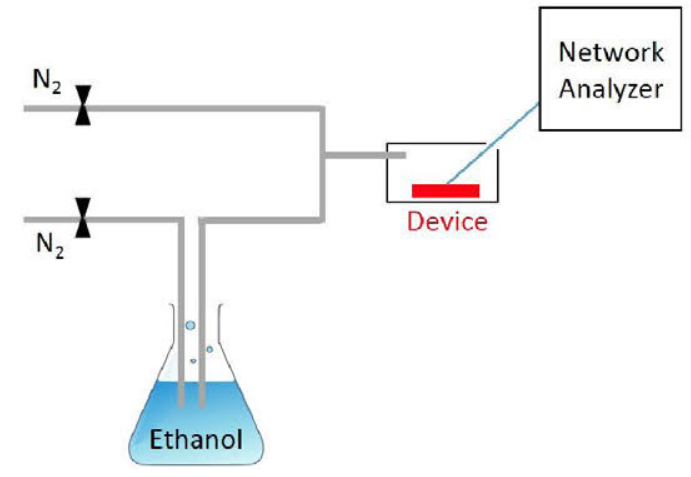

Figure 5: Bubbling system using a $\mathrm{N}_{2}$ carrier to generate ethanol vapors. The device is mounted in a chamber with a small hole through which electrical contact with a network analyzer is achieved.

\section{RESULTS}

The three defined resonant frequencies were monitored at the same time by tracking the maximum of the real part of the admittance $(\operatorname{Re}(\mathrm{Y}))$ curve through a specifically designed LabView application. The procedure was based on acquiring $\operatorname{Re}(\mathrm{Y})$ around the resonant frequency with a bandwidth of $20 \mathrm{MHz}(\sim 4 \mathrm{kHz}$ frequency spacing using 5000 data points) and fitting it to a rational function to accurately measure the frequency at the maximum, which was achieved by deriving the function and calculating the zero cross. Figure 6 shows the evolution of all frequencies upon introduction in the chamber of different ethanol concentrations.

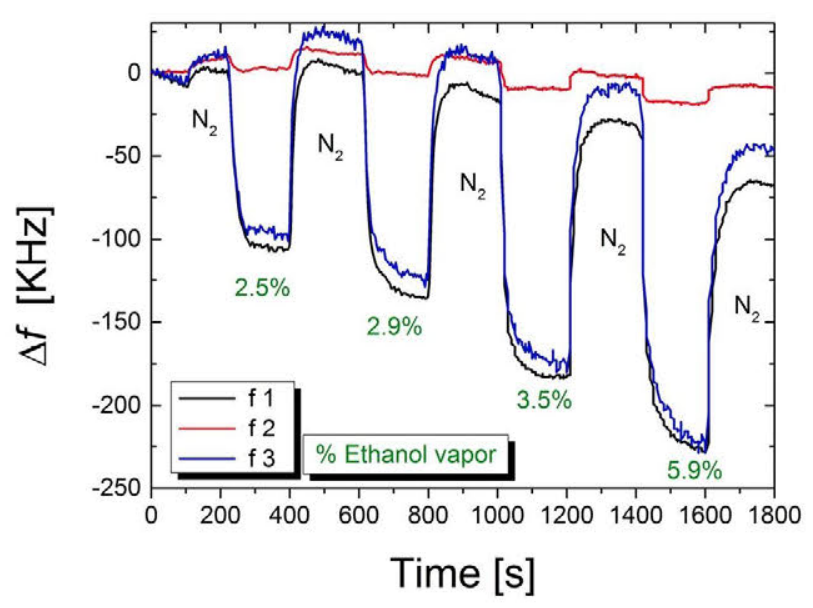

Figure 6: Response of the SMR (shifts in resonant frequency) towards different ethanol concentrations. Before changing the concentration, the chamber was purged with $\mathrm{N}_{2}$ until the resonant frequencies were almost recovered. 
First of all, we observe the pronounced difference in frequency shifts between $f_{1}$ and $f_{3}$ (with CNTs) and $f_{2}$ (without CNTs). These larger shifts of $f_{1}$ and $f_{3}$ upon ethanol introduction confirm that CNTs efficiently adsorb ethanol molecules, which produces a significant increase of the mass deposited over the CNT-covered areas. Moreover, after $\mathrm{N}_{2}$ purging, the CNTs desorb the ethanol molecules until the resonances recover their initial values. However, the observed shift to lower frequencies of the complete curve indicates that the desorption is not fully achieved after several ethanol cycles. In contrast, $f_{2}$ undergoes considerably smaller shifts due to the absence of CNTs that act as sensing elements. We attribute these small shifts only to the humidity in the chamber, which varies with different ethanol concentrations. The result is the condensation of a thin liquid layer on the top electrode that shifts in a smaller proportion the resonant frequency. This humidity affects all three resonances, however mass detection predominates for $\mathrm{f}_{1}$ and $\mathrm{f}_{3}$. For a more accurate mass detection, the response of $f_{2}$ can be extracted from the response of $f_{1}$ and $f_{3}$, resulting only in the response due to mass attachment to the CNTs.

\section{CONCLUSION}

We have proposed a thin film electroacoustic resonator with integrated CNT forests for VOC detection and humidity discrimination. This discrimination can be achieved by defining different zones within the same device with different resonances by pad-regrowth. While one part of the devices has integrated CNT forest the other is left bare. This allows adsorption of VOC molecules only by the CNTs, hence increasing mass and shifting the corresponding resonant frequency, while the bare zone is affected only by the ambient humidity.

\section{ACKNOWLEDGEMENTS}

The authors would like to deeply acknowledge late Prof. Enrique Iborra for his strong support and hard work leading the GMME during all these years.

\section{REFERENCES}

[1] B. Szulczyński, J. Gębicki, B. Szulczyński, and J. Gębicki, "Currently Commercially Available Chemical Sensors Employed for Detection of Volatile Organic Compounds in Outdoor and Indoor Air," Environments, vol. 4, no. 1, p. 21, Mar. 2017.

[2] I. Sayago, M. J. Fernández, J. L. Fontecha, M. C. Horrillo, C. Vera, I. Obieta, and I. Bustero, "New sensitive layers for surface acoustic wave gas sensors based on polymer and carbon nanotube composites," Sensors Actuators B Chem., vol. 175, pp. 67-72, Dec. 2012.

[3] C. Viespe and C. Grigoriu, "Surface acoustic wave sensors with carbon nanotubes and $\mathrm{SiO} 2 / \mathrm{Si}$ nanoparticles based nanocomposites for VOC detection," Sensors Actuators B Chem., vol. 147, no. 1, pp. 43-47, May 2010.

[4] S. Rey-Mermet, R. Lanz, and P. Muralt, "Bulk acoustic wave resonator operating at $8 \mathrm{GHz}$ for gravimetric sensing of organic films," Sensors Actuators B Chem., vol. 114, no. 2, pp. 681-686,
Apr. 2006.

[5] Y. Chang, N. Tang, H. Qu, J. Liu, D. Zhang, H. Zhang, W. Pang, and X. Duan, "Detection of Volatile Organic Compounds by Self-assembled Monolayer Coated Sensor Array with Concentrationindependent Fingerprints.," Sci. Rep., vol. 6, p. 23970, Apr. 2016.

[6] X. Zhang, B. Gao, A. E. Creamer, C. Cao, and Y. Li, "Adsorption of VOCs onto engineered carbon materials: A review," J. Hazard. Mater., vol. 338, pp. 102-123, Sep. 2017.

[7] M. Penza, P. Aversa, G. Cassano, D. Suriano, W. Wlodarski, M. Benetti, D. Cannatà, F. Di Pietrantonio, and E. Verona, "Thin-film bulkacoustic-resonator gas sensor functionalized with a nanocomposite Langmuir-Blodgett layer of carbon nanotubes," IEEE Trans. Electron Devices, vol. 55, no. 5, pp. 1237-1243, 2008.

[8] T. Mirea, M. DeMiguel-Ramos, M. Clement, J. Olivares, E. Iborra, V. Yantchev, and I. Katardjiev, "AlN solidly mounted resonators for high temperature applications," in 2014 IEEE International Ultrasonics Symposium, 2014, pp. 1524-1527.

[9] J. Olivares, T. Mirea, B. Díaz-Durán, M. Clement, M. DeMiguel-Ramos, J. Sangrador, J. De Frutos, and E. Iborra, "Growth of carbon nanotube forests on metallic thin films," Carbon N. Y., vol. 90, 2015.

[10] T. Mirea, J. Olivares, M. Clement, J. Sangrador, and E. Iborra, "Direct integration of CNT forests on solidly mounted resonators and their influence on device performance," in 2017 Joint Conference of the European Frequency and Time Forum and IEEE International Frequency Control Symposium (EFTF/IFC), 2017, pp. 353-356.

\section{CONTACT}

*T. Mirea, tel: +34910672245; teona@etsit.upm.es 